\title{
Erratum to: Chemical Mediators of Inflammation and Resolution in Post-Operative Abdominal Aortic Aneurysm Patients
}

\author{
Padmini S. Pillai, ${ }^{1}$ Stanley Leeson, ${ }^{2}$ Timothy F. Porter, ${ }^{1}$ Christopher D. Owens, ${ }^{3}$ Ji Min Kim, ${ }^{3}$ \\ Michael S. Conte, ${ }^{3}$ Charles N. Serhan, ${ }^{1,4,5}$ and Simon Gelman ${ }^{1,2}$
}

\section{Erratum to: Inflammation \\ DOI 10.1007/s10753-011-9294-8}

The original version of this article contained errors in the structure insets in Fig. 4, Panels b and c and Fig. 5, Panels $\mathrm{b}$ and $\mathrm{c}$. The correct versions of these figures are below.
The online version of the original article can be found at http://dx.doi. org/10.1007/s10753-011-9294-8

${ }^{1}$ Center for Experimental Therapeutics and Reperfusion Injury, Harvard Institutes of Medicine, Brigham and Women's Hospital and Harvard Medical School, Boston, MA 02115, USA

${ }^{2}$ Department of Anesthesiology, Perioperative and Pain Medicine, Brigham and Women's Hospital and Harvard Medical School, Boston, MA 02115, USA

${ }^{3}$ Division of Vascular and Endovascular Surgery, Brigham and Women's Hospital and Harvard Medical School, Boston, MA 02115, USA

${ }^{4}$ Director, Center for Experimental Therapeutics and Reperfusion Injury, Department of Anesthesiology, Perioperative and Pain Medicine, Brigham and Women's Hospital and Harvard Medical School, Harvard Institutes of Medicine, Room 829, 77 Avenue Louis Pasteur, Boston, MA 02115, USA

${ }^{5}$ To whom correspondence should be addressed at Director, Center for Experimental Therapeutics and Reperfusion Injury, Department of Anesthesiology, Perioperative and Pain Medicine, Brigham and Women's Hospital and Harvard Medical School, Harvard Institutes of Medicine, Room 829, 77 Avenue Louis Pasteur, Boston, MA 02115, USA. E-mail: cnserhan@zeus.bwh.harvard.edu
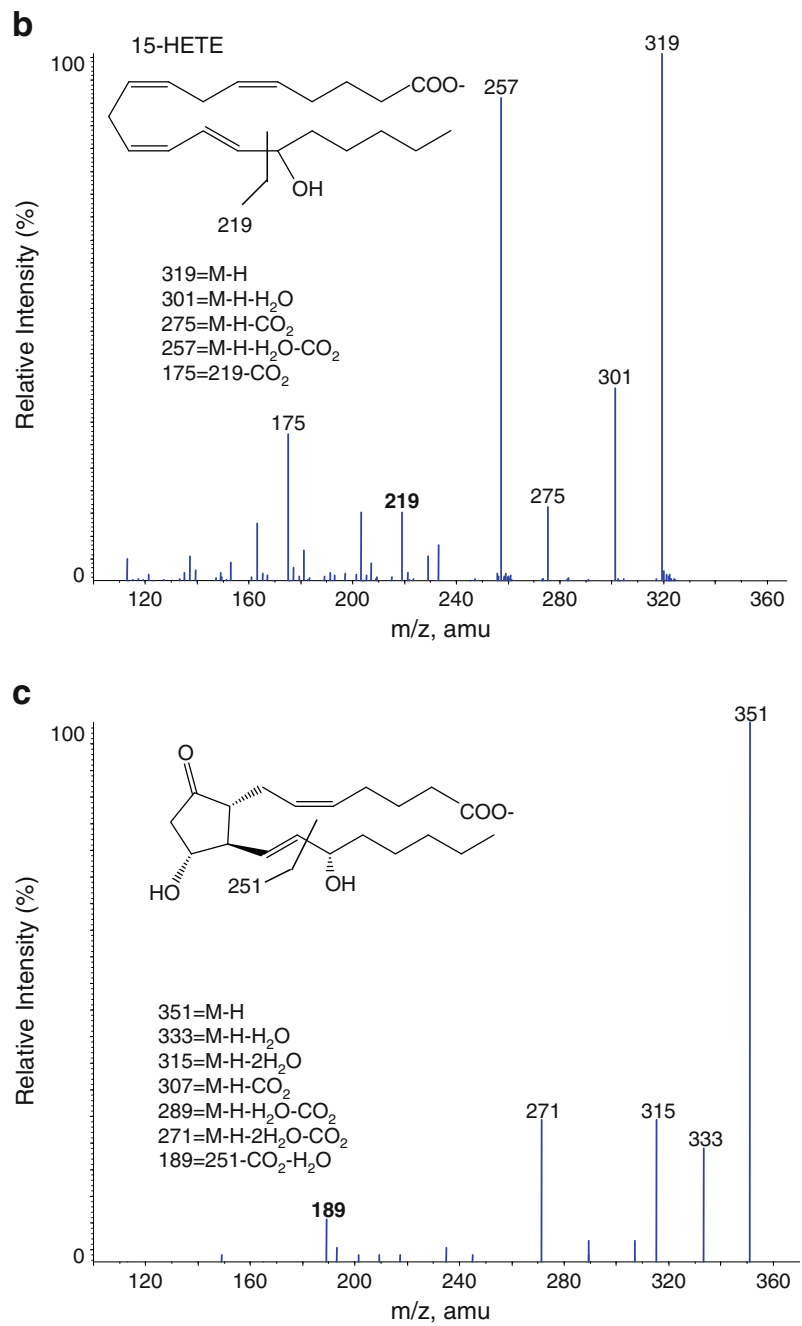

Fig. 4. Representative mass spectra (MS-MS) are shown $(n=4-6)$ for b $15-\mathrm{HETE}$ and $\mathbf{c} \mathrm{PGE}_{2}$. 

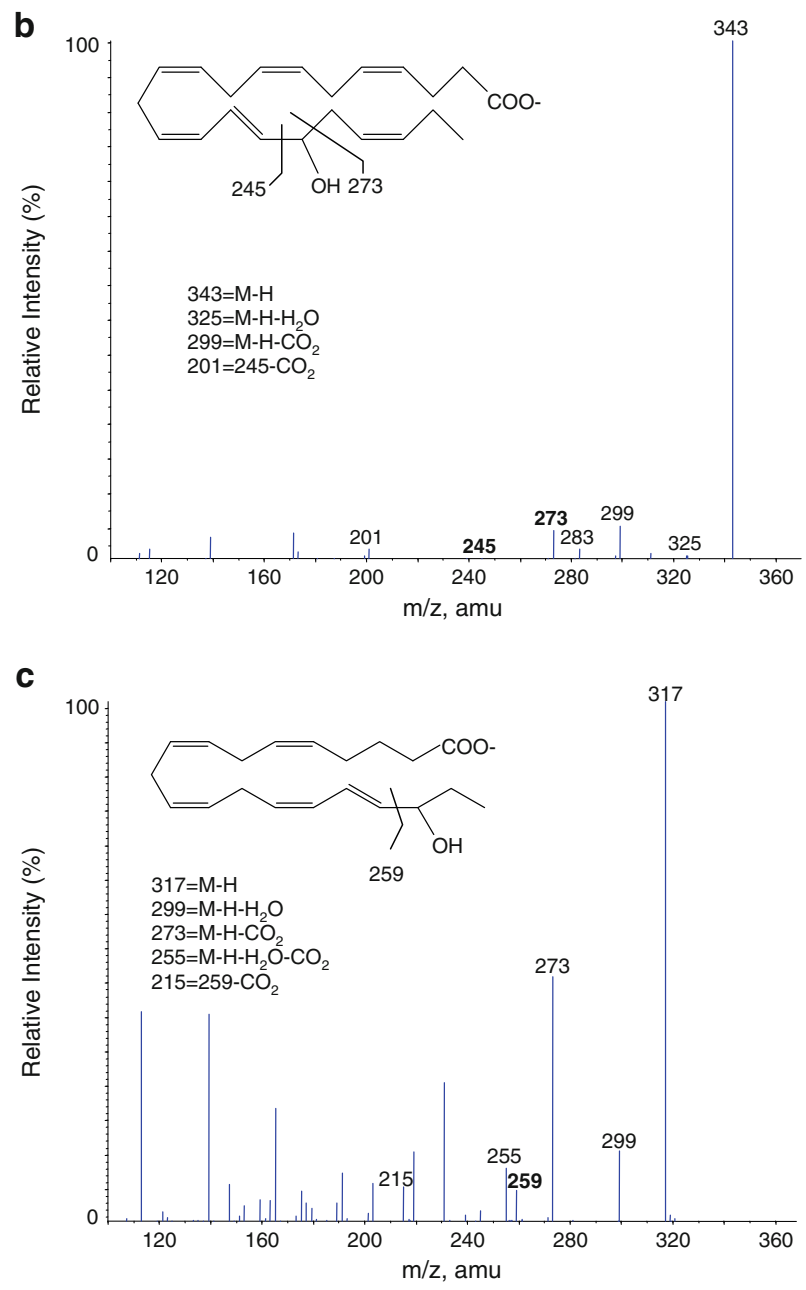

Fig. 5. Representative mass spectra $(n=4-6)$ are shown for b $17-\mathrm{H}-$ DHA and $\mathbf{c} 18$-HEPE. 